\title{
Design of Nonlinear Controllers for an Electrohydraulic Velocity Servo-System
}

\author{
Saleh Alshamali and Mohamed Zribi \\ Department of Electrical Engineering \\ Kuwait University, P. O. Box 5969, Safat, 13060, Kuwait \\ s.alshamali@ku.edu.kw
}

\begin{abstract}
This paper addresses the control problem of an electrohydraulic velocity servo-system (EHVSS). Two control schemes are proposed for the stabilization of the system. The first scheme is a dynamic sliding mode controller (SMC), and the second scheme is a linear quadratic regulator-like control law which is based on the state-dependent Riccati equation (SDRE) approach. When applying the SMC technique, the mathematical model of the system is transformed into the controller canonical form via a nonlinear transformation to facilitate the control design. When applying the SDRE technique, the EHVSS dynamics are parameterized to produce a system with a state-dependent coefficient matrix; then the linear quadratic controller technique is carried out to produce a sub-optimal state feedback control law that regulates the system trajectories to their desired values. The effectiveness of the proposed controllers is validated through computer simulations.
\end{abstract}

Keywords: Nonlinear Control, Electrohydraulic servo-system, State-Dependent Riccati Equation, sliding mode control

\section{Introduction}

Electrohydraulic servo systems can be found in several industrial applications such as commercial aircrafts, construction equipments and lift controls [1,2]. These systems are characterized by high power to weight ratio, fast response, and high stiffness [3]. Electrohydraulic servos come in different types. The most common types are the position servo, the velocity servo, and the force servo. This work is concerned with the velocity (speed) control of the electrohydraulic servo system where a transducer connected to the output measures the velocity. The system description and the mathematical model are discussed in the ensuing sections.

Several control strategies were proposed for the position, speed, pressure, and force control of electrohydraulic servo systems (EHSS). The contributions of some of these works are summarized in this section. In [4], the feedback linearization and the backstepping control schemes are used in the velocity control of an electrohydraulic servo system. The controllers are shown to drive the state trajectories to their desired set points. An indirect adaptive backstepping control law is introduced in [5] for the position control of an electrohydraulic servo system under parameter variations. The proposed controller is shown to compensate for the system's parameters, while ensuring the stability of the closed-loop system. In [6], a feedback linearization controller is proposed for the position control of an electrohydraulic servo system under supply pressure variations; a switching control law is developed to account for the uncertainty in the supply pressure. The work in [7] develops a combined sliding mode controller and a fuzzy radial basis function neural network controller to stabilize an electrohydraulic servo system; the proposed control law is a function of the sliding surface and the radial basis function network parameters. In [8], a robust backstepping controller for the EHSS subject to un-modeled 
dynamics and parametric uncertainty is introduced. First, a state transformation is used to convert the system dynamics into a strict feedback form, then a robust controller is utilized to derive the control law. In [9], an adaptive feedback linearizing controller is introduced for the speed control of an electrohydraulic servo system. Its performance is compared to that of the indirect adaptive fuzzy controller, where it is shown that the adaptive fuzzy controller produced a better response. The work in [10] uses the generalized predictive controller methodology, along with a recursive technique to estimate the system parameters, for the force control of an electrohydraulic servo system. The controller is implemented on an actual unit and it is shown to have good tracking and regulation properties. The stabilization problem of an electrohydraulic servo system via a radial basis function neural network-based variable structure controller is tackled in [11]. The augmented control law is shown to reduce the effects of the uncertainties and to improve the tracking of the trajectories of the system. In [12], a sliding mode controller is designed for the reference tracking of an electrohydraulic servo system; a fuzzy logic technique is used to tune the parameters of the controllers.

This paper deals with the stabilization of an electrohydraulic velocity servo system (EHVSS). At first, a sliding mode controller is proposed to stabilize the EHVSS. The design of a sliding mode controller is a two-stage process. First, a switching surface (also known as the sliding manifold) is defined such that the system achieves its desired properties once confined to that surface. Next, a nonlinear switching control law is designed to force the system trajectories to the sliding surface in finite time, and to maintain them there for all subsequent time [13]. When using this controller, a nonlinear transformation is introduced to render the nonlinear system into the controller canonical form. Then a dynamic SMC is designed to stabilize the system.

In the second part of the paper, the SDRE approach is used to stabilize the EHVSS. The original electrohydraulic dynamics are first parametrized into a special form such that the system matrix is state-dependent. Then, an LQR controller is applied at each sampling instance to the parameterized system [14]. The implementation of the SDRE method is simple and allows for a tradeoff between the state errors and the control action. Furthermore, since the parameterization is not unique, the SDRE approach has an extra degree of freedom to improve the control design [15].

The rest of the paper is organized as follows. The model of the electrohydraulic velocity servo system is described in Section 2; this model is then transformed into a form that facilitates the design of nonlinear control schemes. The dynamic sliding mode controller is proposed in Section 3. The application of the SDRE approach to the EHVSS system is discussed in Section 4. In Section 5, simulation studies of the performance of the proposed controllers are presented, discussed, and compared. Finally, some concluding remarks are given in Section 6.

For convenience, the arguments of a function will be omitted in the analysis when no confusion may arise.

\section{Dynamic Model of the System}

The electrohydraulic velocity servo system (EHVSS) under consideration is shown in Figure 1. The main components of an EHVSS are: a hydraulic power supply, an accumulator, a charge valve, a pressure gauge device, a filter, a two-stage electro-hydraulic servo-valve, a hydraulic motor, a measurement device, a computer, and a voltage-to-current converter. 


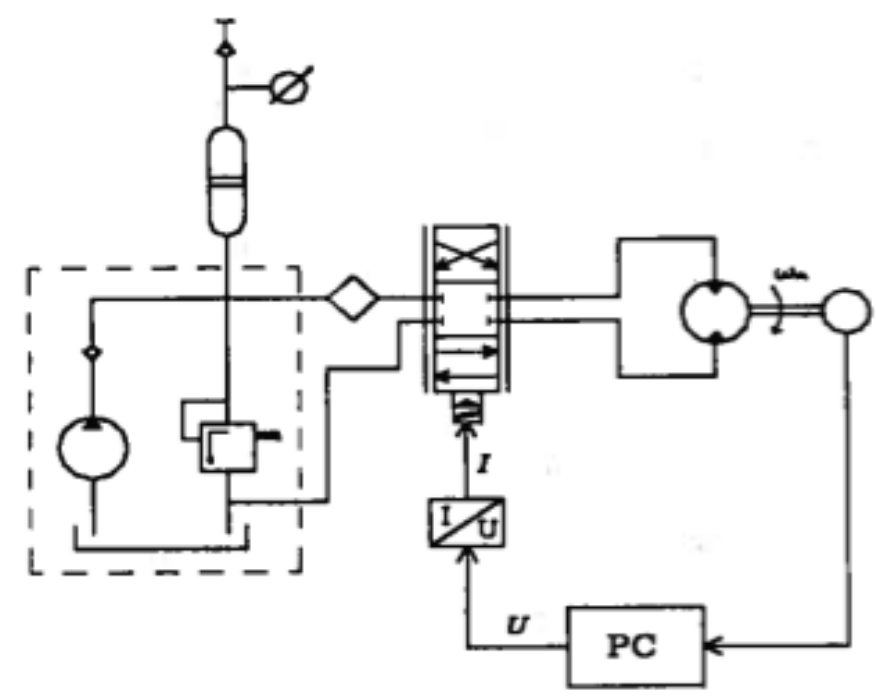

Figure 1. Electrohydraulic Velocity Servosystem [4]

The basic operation of an electrohydraulic velocity servo system can be summarized as follows: a transducer measures the output of the servo and converts it into an electrical signal (voltage). The difference between this signal and the command reference is fed to an amplifier which in turn produces the signal necessary to turn the valve. The valve movement, then, allows a specific amount of fluid to run the actuator, and hence control the hydraulic motor velocity [16].

\subsection{The Mathematical Model of the EHVSS}

The equations describing a third order model of an electro-hydraulic velocity servo-system are adopted from [4] such that:

$$
\begin{aligned}
& \dot{x}_{1}=\frac{1}{J}\left(-B_{m} x+q x-q C{ }_{s} P s(i g)\right. \\
& \dot{x}_{2}=\frac{2 \beta_{e}}{V_{o}}\left(-q_{n} x-C_{m} x+C W \sqrt{\frac{1}{x_{3}}\left({ }_{s} P{ }_{2} x \leqslant i g\right)}\right. \\
& \dot{x}_{3}=\frac{1}{T_{r}}\left(-x_{3}+\frac{K_{r}}{K_{q}} u\right) \\
& y=x_{1}
\end{aligned}
$$

where the states of the system are: $x_{1}(t)$, the motor angular velocity $[\mathrm{rad} / \mathrm{s}], x_{2}(t)$, the load pressure velocity $[\mathrm{Pa}]$, and $x_{3}(t)$, the valve displacement $[m]$. The state vector is $x=\left[\begin{array}{lll}x_{1} & x_{2} & x_{3}\end{array}\right]^{T}$, the control variable is $u$. The output of the system, $y$, is the motor angular velocity. The definitions and the nominal values of the system parameters in (2.1) are given in Table 1.

\section{Table 1. Parameters of the Electrohydraulic Velocity Servo System}

\begin{tabular}{lll} 
The parameter & Definition & Nominal value \\
\hline$J$ & $\begin{array}{l}\text { Total Inertia of the motor and the load referred to } \\
\text { motor shaft }\end{array}$ & $0.03 \mathrm{kgm}^{2}$
\end{tabular}




\begin{tabular}{lll}
$q_{m}$ & Volumetric displacement of the motor & $7.96 \times 10^{-7} \mathrm{~m}^{3} / \mathrm{rad}$ \\
$B_{m}$ & Viscous damping coefficient & $1.1 \times 10^{-3} \mathrm{Nms}$ \\
$\beta_{e}$ & Effective bulk modulus of the system & $1.391 \times 10^{-4} \mathrm{~Pa}$ \\
$C_{f}$ & Dimensionless internal friction coefficient & 0.104 \\
$V_{o}$ & Average contained volume of each motor & $1.2 \times 10^{-4} \mathrm{~m}^{3}$ \\
$C_{d}$ & chamber & 0.61 \\
$C_{i m}$ & Dimensionless discharge coefficient & $1.69 \times 10^{-11} \mathrm{~m}^{3} /($ Pa.s $)$ \\
$P_{s}$ & Cross-port leakage coefficient of the motor & $10^{7} \mathrm{~Pa}$ \\
$\rho$ & Supply pressure & $850 \mathrm{~kg} / \mathrm{m}^{3}$ \\
$T_{r}$ & Oil density & $0.01 \mathrm{~s}$ \\
$K_{r}$ & Valve time constant & $1.4 \times 10^{-4} \mathrm{~m}^{3} / \mathrm{sV}$ \\
$K_{q}$ & Valve gain & $1.66 \mathrm{~m}^{2} / \mathrm{s}$ \\
$W$ & Valve flow gain & $8 \pi \times 10^{-3} \mathrm{~m}$ \\
\hline
\end{tabular}

The dynamics model in (2.1) can be simplified by adopting the following practical assumptions ([4], [7]):

Assumption 1 The motor angular velocity, $x_{1}>0$, i.e., the motor shaft does not change its direction of rotation.

This requires the next assumption.

Assumption 2 The servo valve displacement, $x_{3}>0$, i.e., the valve moves in one direction relative to its neutral position.

Under these two assumptions, the model (2.2) reduces to the following:

$$
\begin{aligned}
& \dot{x}_{1}=\frac{1}{J}\left(-B_{m} x+q{ }_{2} x-{ }_{m} q C\right. \\
& \dot{x}_{2}=\frac{2 \beta_{e}}{V_{o}}\left(-q_{n} x-C_{m} x+C \sqrt{\frac{1}{\rho}\left({ }_{s} P\right.}{ }_{2}\right. \\
& \dot{x}_{3}=\frac{1}{T_{r}}\left(-x_{3}+\frac{K_{r}}{K_{q}} u\right) \\
& y=x_{1}
\end{aligned}
$$

Remark 1: Note that from the practical point of view, the supply pressure is always larger than the load pressure velocity, i.e., $P_{s}>x_{2}$.

The objective of the paper is to design controllers to steer the state trajectories of the EHVSS to the desired constant operating points. To this end, let the vector of desired states be defined as $x_{d}=\left[\begin{array}{lll}x_{1 d} & x_{2 d} & x_{3 d}\end{array}\right]^{T}$. Also, let the control input which enables the system to achieve the desired states be denoted by $u_{d}$ such that $u_{d}=\frac{k_{q}}{k_{r}} x_{3 d}$. Using the 
dynamics of the system (2.2) at steady-state, it can be shown that the desired states $x_{1 d}$, $x_{2 d}$, and $x_{3 d}$, satisfy the following equations:

$$
\begin{aligned}
& x_{1 d} \quad \text { chosen v: } \\
& x_{2 d}=\frac{B_{m}}{q_{m}} x_{1 d}+C_{f} P_{s} \\
& x_{3 d}=\frac{1}{C_{d} W \sqrt{\frac{1}{\rho}\left(P_{s}-x_{2 d}\right)}}\left(q_{m} x_{d d}+C_{i m} x_{d}\right.
\end{aligned}
$$

\subsection{State Transformation of the Model}

It is obvious from eqn. (2.2) that the equations describing the servo system are highly nonlinear. Therefore, to facilitate the design of nonlinear control schemes, a change of variables is proposed.

Consider the change of variables $z(t)=T(x)$, with $z(t)=\left[\begin{array}{lll}z_{1}(t) & z_{2}(t) & z_{3}(t)\end{array}\right]^{T}$ such that,

$$
\begin{aligned}
z_{1}= & x_{1}-x_{d 1}=y-y_{d} \\
z_{2}= & \frac{1}{J}\left(-B_{m} x+q{ }_{2} x-{ }_{m} q C\right. \\
z_{3}= & \frac{-B_{m}}{J^{2}}\left(-B_{m} x+q{ }_{2} x-{ }_{m} q C\right. \\
& +\frac{2 \beta q_{m}}{J V_{o}}\left(-q_{m} x-C_{c_{2} 2} C_{d} \sqrt[W]{\frac{1}{\rho}\left({ }_{s} P_{2}\right)}\right. \\
= & \left.p_{1} x_{1}+p_{2} x_{2}+p_{3} \sqrt{\frac{1}{x_{3}}\left({ }_{s} P\right.}\right) x_{2}+
\end{aligned}
$$

where the parameters $p_{1}, p_{2}, p_{3}$, and $p_{4}$ are defined such that $p_{1}=\frac{B_{m}^{2}}{J^{2}}-\frac{2 \beta_{e} q_{m}^{2}}{J V_{o}}$, $p_{2}=\frac{-B_{m} q_{m}}{J^{2}}-\frac{2 \beta_{e} q_{m} C_{i m}}{J V_{o}}, \quad p_{3}=\frac{2 \beta_{e} q_{m} C_{d} W}{J V_{o}}$, and $p_{4}=\frac{B_{m} q_{m} C_{f} P_{s}}{J^{2}}$.

Using the system dynamics (2.2), and the state transformation (2.4), the dynamics of the electro-hydraulic velocity servo system in the new coordinates can be expressed as follows:

$$
\begin{aligned}
& \dot{z}_{1}=z_{2} \\
& \dot{z}_{2}=z_{3} \\
& \dot{z}_{3}=f_{1}(x)+g(x) u=f(+z) \quad g( \\
& y=z_{1}+x_{1 d}
\end{aligned}
$$

where 


$$
\begin{gathered}
f_{1}=\frac{p_{1}}{J}\left(-B_{m} x_{1}+q_{m} x_{2}-q_{m} C_{f} P_{s}\right)-\frac{p_{3}}{T_{r}} x_{3} \sqrt{\frac{1}{\rho}\left(P_{s}-x_{2}\right)} \\
+\frac{2 \beta_{e}}{V_{o}}\left(p_{2}-\frac{1}{2 \rho} \frac{1}{\sqrt{\frac{1}{\rho}\left(P_{s}-x_{2}\right)}} p_{3} x_{3}\right) \times+q_{m} x{ }_{1} C_{i m} x+{ }_{2} C_{d} W x \sqrt{\frac{1}{\rho} P_{s}-x} \\
g_{1}=\frac{p_{3}}{T_{r}} \frac{K_{r}}{K_{q}} \sqrt{\frac{1}{\rho}\left(P_{s}-x_{2}\right)}
\end{gathered}
$$

Remark 2: From (2.3)-(2.4), it is easy to check that if $z(t)$ converges to zero as $t \rightarrow \infty$ , then $x(t)$ converges to $x_{d}$ as $t \rightarrow \infty$.

\section{Design of a Dynamic Sliding Mode Controller}

In this section, a dynamic sliding mode control law is designed for the electrohydraulic velocity servo-system. The design is motivated by the work in [17]. Dynamic SMC adds additional dynamics to the system such that the augmented system is a combination of the original system plus an integrator [18]. It, therefore, has the advantage of reducing the chattering problem associated with classical SMC design, and it helps improve the overall performance of the system [19].

To this end, let the scalars $\beta_{1}, \beta_{2}$ and $\beta_{3}$ be real positive constants such that the polynomial $P_{2}(s)=s^{3}+\beta_{3} s^{2}+\beta_{2} s+\beta_{1}$ is Hurwitz. Also, define the input-dependent switching surface $\sigma_{2}(z, u)$ such that,

$$
\sigma_{2}(z, u)=l t \frac{1}{g_{1}} \quad\left(f+\beta_{1} \quad z+\beta_{2} z_{2}+\beta_{3}\right.
$$

Let $\eta_{2}$ be a sufficiently large, strictly positive scalar. The following proposition gives the main result of this section.

Proposition 2: The dynamic sliding mode control scheme:

$$
u=-\frac{1}{g_{1}}\left(f_{1}+\beta_{1} z_{1}+\beta_{2} z_{2}+\beta \quad z\right)_{3}+
$$

with

$$
\dot{v}=-\eta_{2} \text { sig } g\left(n+\frac{1}{g_{1}}\left({ }_{1}+f \beta_{1}+z \beta_{2}+{ }_{2} z \beta_{3}\right)\right)
$$

when applied to the electrohydraulic velocity servo-system (2.5), guarantees the asymptotic convergence of $z_{1}(t), z_{2}(t)$ and $z_{3}(t)$ to zero as $t \rightarrow \infty$.

Proof: Taking the derivative of (3.1) with respect to time yields, 


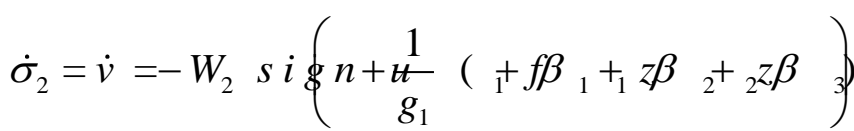

$$
\begin{aligned}
& =-\eta_{2} \operatorname{sign}\left(\sigma_{2}\right)
\end{aligned}
$$

The trajectories associated with the discontinuous dynamics (3.4) exhibit a finite time reachability to zero from any given initial condition provided that the scalar $\eta_{2}$ is chosen to be strictly positive. Moreover, the dynamics (3.4) guarantees that $\sigma_{2} \dot{\sigma}_{2}=-\eta_{2}\left|\sigma_{2}\right|<0$, which indicates that the sliding surface is reached in finite time.

Since $\sigma_{2}$ is driven to zero in finite time, then the closed-loop system is governed on the sliding surface $\sigma_{2}=0$ by the following equation:

$$
\dot{z}(t)=A_{c 2} \quad z(
$$

where

$$
A_{c 2}=\left[\begin{array}{ccc}
0 & 1 & 0 \\
0 & 0 & 1 \\
-\beta_{1} & -\beta_{2} & -\beta_{3}
\end{array}\right]
$$

The solution of (3.5) is $z(t)=e^{A_{c 2} t} z(0)$. Since $A_{c 2}$ is a stable matrix due to the choice of the parameters $\beta_{i}(i=1,2,3)$, then $z(t)$ converges to zero as $t \rightarrow \infty$. Thus, $z_{1}(t), z_{2}(t)$ and $z_{3}(t)$ converge to zero as $t \rightarrow \infty$.

Thus, it can be concluded that the dynamic sliding mode controller (3.2)-(3.3) when applied to the electrohydraulic velocity servo-system (2.5), guarantees the asymptotic convergence of $z_{1}(t), z_{2}(t)$ and $z_{3}(t)$ to zero as $t \rightarrow \infty$. Moreover, the controller (3.2)-(3.3) when applied to the electrohydraulic velocity servo-system (2.2) guarantees the asymptotic convergence of $x_{1}(t), x_{2}(t), x_{3}(t)$ to their desired values as $t \rightarrow \infty$.

\section{Design of a Controller Using the SDRE Approach}

In this section, the state-dependent Riccati equation (SDRE) approach is used in the design of a controller for the electrohydraulic velocity servo system. The idea of the SDRE approach can be summarized as follows [20]. Given a nonlinear system of the form:

$$
\dot{x}(t)=f(x+) \quad g(x
$$

where $x \in \mathfrak{R}^{n}$ and $f: \mathfrak{R}^{n} \rightarrow \mathfrak{R}^{n}$.

First, the system (4.1) is parametrized into the following form:

$$
\dot{x}(t)=A(x)+x \quad B(:
$$

where $A(x): \mathfrak{R}^{n} \rightarrow \mathfrak{R}^{n \times n}, B(x): \mathfrak{R}^{n} \rightarrow \mathfrak{R}^{n \times m}$, and $u \in \mathfrak{R}^{m}$. It should be noted that the parameterization producing the state-dependent coefficient (SDC) matrix $A(x)$ is not unique. 
The objective of the SDRE method is to minimize the cost function

$$
J_{c}=\frac{1}{2} \int_{0}^{\infty}\left(x^{T} Q x+u^{T} R u\right) d t
$$

where the weighting matrices $Q$ and $R$ are such that $Q \geq 0$, and $R>0$.

Next, the well-known linear quadratic regulator (LQR) controller is applied to the system (4.2) on a point-wise basis. Furthermore, at each sampling instant, the SDC matrix $A(x)$ is treated as a constant matrix.

Then, the obtained linear feedback regulator is given by:

$$
u=-K(x) x=-R^{T} B(x) P
$$

where $P(x)$ is the symmetric, positive-definite solution of the stated-dependent Riccati equation:

$$
A^{T}(x) P(x)+P(x) A(x)-P(x) B(x) R^{-1}(x) B^{T}(x) P(x)+Q(x)=0
$$

Notice that the SDRE method requires that all the states be measurable, and that the pair ( $A(x), B(x)$ ) be point-wise controllable [21].

It should be kept in mind that the LQR technique leads to a regulation to the origin. Therefore, before applying this technique to the Electrohydraulic servo-system, a change of variables is introduced such that the origin is the new equilibrium point. The EHVSS dynamics, given in Eqn. (2.2), are first re-written by defining the $a_{i}$ parameters (

\begin{tabular}{|c|c|c|c|c|c|c|c|}
\hline Parameter & $a_{1}$ & $a_{2}$ & $a_{3}$ & $a_{4}$ & $a_{5}$ & $a_{6}$ & $\begin{array}{ll}a_{7} & a_{8}\end{array}$ \\
\hline \multirow[t]{2}{*}{ Value } & $-B_{m}$ & $\underline{q_{m}}$ & $-q_{m} C_{f} P_{s}$ & $-2 \beta_{e} q_{m}$ & $-2 \beta_{e} C_{i m}$ & $2 \beta_{e} C_{d} W$ & $\underline{-1}$ \\
\hline & $J$ & $\bar{J}$ & $J$ & $V_{o}$ & $V_{o}$ & $V_{o}$ & $\overline{T_{r}} \overline{T_{r} K_{q}}$ \\
\hline
\end{tabular}
$i=1,2, \cdots, 8)$ as shown in Table 2 .

Table 2. The $a_{i}(i=1,2,3)$ parameters of the EHVSS

The EHVSS model is then written as follows:

$$
\begin{aligned}
& \dot{x}_{1}=a_{1} x_{1}\left(\text { t) } \quad a_{2} x_{2}(+y)\right. \\
& \dot{x}_{2}=a_{4} x_{1}\left(\text { t) } \quad a_{5} x_{2}(t) \quad q \sqrt{\frac{1}{\frac{x}{\rho}}{ }_{s}(-P}\right. \\
& \dot{x}_{3}=a_{7} x_{3}+a_{8} \\
& y=x_{1}
\end{aligned}
$$

Next, define the error vector $\tilde{x}(t)=\left[\begin{array}{lll}\tilde{x}_{1}(t) & \tilde{x}_{2}(t) & \tilde{x}_{3}(t)\end{array}\right]^{T}$ such that $\tilde{x}_{1}=x_{1}-x_{1 d}$, $\tilde{x}_{2}=x_{2}-x_{2 d}$, and $\tilde{x}_{3}=x_{3}-x_{3 d}$. Taking the time-derivative of $\tilde{x}(t)$, the dynamics (4.3) can be written as, 


$$
\begin{aligned}
\dot{\tilde{x}}_{1}= & a_{1} \tilde{x}_{1}(t)+\tilde{a}_{2} x_{2}( \\
\dot{\tilde{x}}_{2}= & a_{4} \tilde{x}_{1}(t)+a \tilde{x}_{5} t_{2}(+) a \bar{x}_{\sqrt{\frac{1}{\rho} P_{s}\left(-x_{d}-\tilde{x}\right.}} \\
& +a_{4} x_{t}+a x_{d 2}+a x_{6 d} \sqrt{\frac{1}{\rho}\left(P_{s}-x_{d}-\tilde{x}\right)} \\
\dot{\tilde{x}}_{3}= & a_{7} \tilde{x}_{3}+a_{8}+t \quad a_{d d}
\end{aligned}
$$

It is clear that as $\tilde{x}(t) \rightarrow 0$ as $t \rightarrow \infty$, then $x(t) \rightarrow x_{d}$ (i.e., the state trajectories converge to their desired values).

Now, the control law is chosen to be of the form

$$
u(t)=\boldsymbol{t}_{s}+u_{e}
$$

where $u_{s}(t)$ is the SDRE controller, and $u_{e}(t)=-a_{7} a_{8} x_{3 d}$ is used to ensure that $\tilde{x}_{3}(t)$ reaches the zero equilibrium at steady-state. Define $f_{\tilde{x}}$ such that

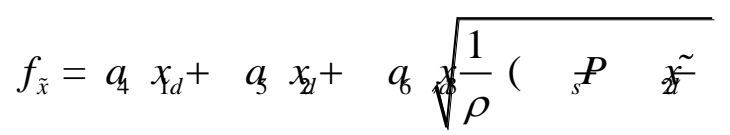

and invoking the expression for $u_{e}(t)$, the system equations (4.4) can be parametrized into the form

$$
\dot{\tilde{x}}(t)=A(\tilde{x}) \tilde{x}+B u
$$

where

$$
A(\tilde{x})=\left[\begin{array}{ccc}
a_{1} & a_{2} & 0 \\
a_{4} & a_{5} & a_{6} \sqrt{\left.(1 / \rho)\left(P_{s}-x_{2 d}-\tilde{x}_{2}\right)\right)}+\frac{f_{\tilde{x}}}{\tilde{x}_{3}} \\
0 & 0 & a_{7}
\end{array}\right] \quad B=\left[\begin{array}{c}
0 \\
0 \\
a_{8}
\end{array}\right] .
$$

It can be shown by using the controllability matrix $C=\left[\begin{array}{ll}B & A(\tilde{x}) B\end{array} A(\tilde{x})^{2} B\right]$ that the system (4.5) is controllable (i.e., $\operatorname{det} C \neq 0$ ) for all values of $\tilde{x}$. Hence, the design of the SDRE-based controller for the EHVSS starts by using the cost function:

$$
J_{c}=\frac{1}{2} \int_{0}^{\infty}\left(\tilde{x}^{T} Q \tilde{x}+u^{T} R u\right) d t
$$

where the weighting matrices $Q \in R^{3 \times 3}$ and $R \in \mathfrak{R}$ are such that $Q \geq 0$, and $R>0$. The state feedback regulator is then given by

$$
u=-K(\tilde{x}) \tilde{x}=-R^{-1} B^{T}(\tilde{x}) P(\tilde{x}) \tilde{x}
$$

where $P(\tilde{x})>0$ is the solution of the following equation: 


$$
A^{T}(\tilde{x}) P(\tilde{x})+P(\tilde{x}) A(\tilde{x})-P(\tilde{x}) B(\tilde{x}) R^{-1}(\tilde{x}) B^{T}(\tilde{x}) P(\tilde{x})+Q(\tilde{x})=0
$$

In this paper, the SDRE problem is solved online using the Matlab software.

Remark 3: For low order systems, an analytic solution of the state-feedback controller can be obtained. However, for high order systems, obtaining an exact solution can be quite difficult.

\section{Simulation Results}

In this section, simulation studies are presented to verify the performance of the proposed controllers. Consider the nonlinear electrohydraulic velocity servo-system (EHVSS) given in (2.2). The system parameters are given in Table 1, and the initial conditions are chosen to be $x(0)=\left[\begin{array}{lll}0 & 0 & 0\end{array}\right]^{T}$. Let the desired motor angular velocity be $x_{1 d}=200 \mathrm{rad} / \mathrm{sec}$. Then, from eqn. (2.3), the corresponding desired values of the load pressure, and the valve displacement are such that $x_{2 d}=1.3164 \times 10^{6} \quad \mathrm{~Pa}$ and $x_{3 d}=1.1710 \times 10^{-4} \mathrm{~m}$, respectively.

The performance of the dynamic sliding mode controller (3.2)-(3.3) is depicted in Figures 2-4. The controller parameters are such that $\beta_{1}=3, \beta_{2}=7, \beta_{3}=4$, and $\eta_{2}=50$. The closed-loop eigenvalues are therefore given by $\lambda_{1}=-0.6074$, $\lambda_{2}=-1.6963+j 1.4359$ and $\lambda_{3}=-1.6963-j 1.4359$, which lead to the controlled system being asymptotically stable. Figure 2 depicts the asymptotic convergence of the motor angular velocity to the desired value $x_{1 d}=200 \mathrm{rad} / \mathrm{sec}$. Figure 3 shows the evolution of the load pressure trajectory towards its desired value; where, after an initial increase, it descends towards the operating point. The smooth convergence of the valve displacement is shown in Figure 4.

Next, the simulation of the SDRE controller is carried out using the weights $Q=\operatorname{diag}\left(6 \times 10^{7}, 0.12,0.3\right)$ and $R=10$. The simulation results of the SDRE controller are presented in Figures 5-7. Figure 5 shows the asymptotic convergence of the motor angular velocity, $x_{1}(t)$, towards the desired value, $x_{1 d}=200 \mathrm{rad} / \mathrm{sec}$. The convergence of the load pressure and valve displacement trajectories towards their desired values is depicted in Figures 6 and 7, respectively. It is clear that both trajectories start with an initial spike before converging to their desired operating points. An inset is provided in Figure 7 to show the initial evolution of the valve displacement trajectory.

From the simulation results, it appears that the dynamic sliding mode controller has a better performance than the static sliding mode controller and the SDRE controller. It converges smoothly towards the operating point, and has less overshoot with respect to the load pressure and valve displacement trajectories. The simulation of the SDRE controller show an initial spike as depicted in Figures 6 and 7. Furthermore, the convergence of the motor velocity, $x_{1}(t)$, towards the desired value is slower under the SDRE controller than under the sliding mode controllers.

Finally, to check the robustness of the proposed controllers, a second set of simulations is carried out as shown in Figures 8-13. The simulations depict the performances of the two proposed controllers, in particular, the motor velocity $x_{1}(t)$ (output) and the control action $u(t)$, when a perturbation term $d(t)$ is added to the first two ODEs of system (2.2). The perturbation term is taken to be $d(t)=e^{-3 t}$. 
The performance of the dynamic SMC in the presence of $d(t)$ is shown Figures 8 and 9. It is clear from Figure 8 that the target value of the motor velocity is reached. Furthermore, the control law is chatter-free as illustrated in inset in Figure 9. For the sake of comparison, a simulation of the system has been performed using the classical SMC as opposed to the proposed dynamic SMC. The result is shown in Figure 10 where it appears, in the inset, that the control law suffers from chattering. The chattering behavior can excite un-modeled dynamics, and can cause the system actuators to wear out. Finally, Figures 11 and 12 depict the performance of the SDRE controller under perturbation. The output trajectory converges to the desired value as shown in Figure 11. However, the control trajectory has a very large initial spike as evident in Figure 12. It can, therefore, be concluded that the control action in the dynamic SMC has better performance than that of the SDRE controller and of the classical SMC because it is chatter-free and has less control effort.

\section{Conclusion}

The regulation problem of an electrohydraulic velocity servo system is considered in this paper. A dynamic sliding mode controller and an SDRE-based controller are proposed for the system. The design of the SMC schemes involves using a nonlinear transformation to transform the system into a canonical form. Asymptotic stability of the closed-loop system is proved using the Lyapunov technique. The design of the SDRE controller involves parametrizing the original dynamics such that the system has a state-dependent coefficient (SDC) matrix. The LQR control scheme is then applied at each sampling instance to the parametrized system. The simulation results show the proposed controllers successfully drive the system trajectories to their desired values. However, the sliding mode controller has better performance than the SDRE controller. In particular, the output trajectory (motor velocity) has faster convergence and less control effort when using the SMC scheme.

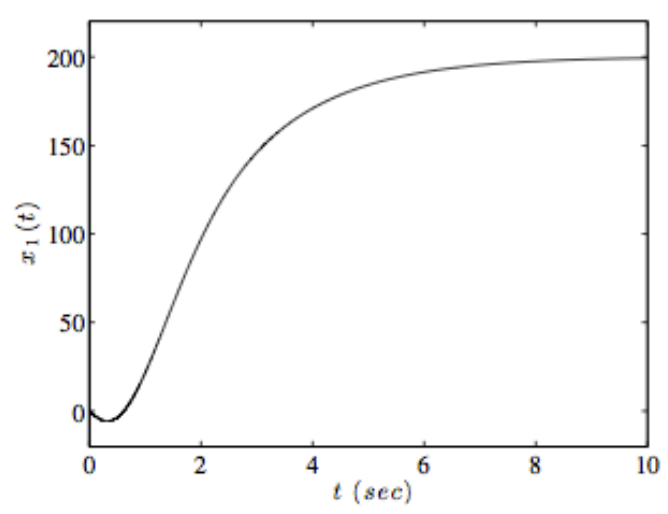

Figure 2. The Motor Velocity $x_{1}(t)$ when Using the Dynamic SMC

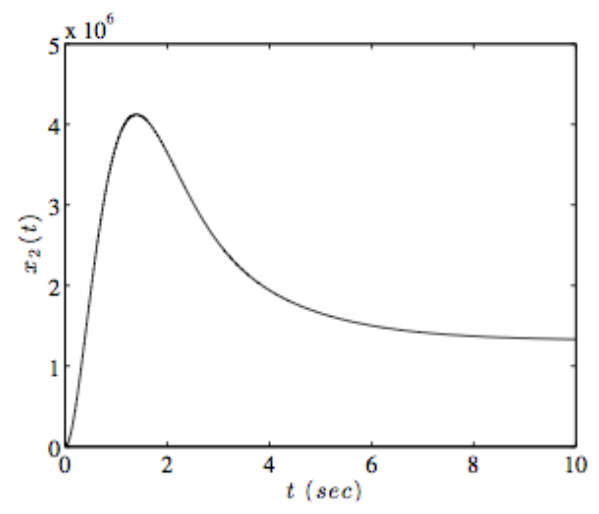

Figure 3. The Load Pressure $x_{2}(t)$ when Using the Dynamic SMC 


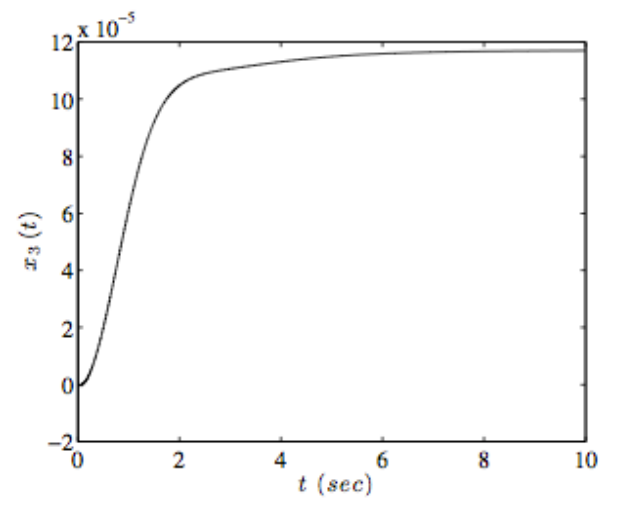

Figure 4. The Valve Displacement $\mathrm{x}_{3}(\mathrm{t})$ when Using the Dynamic SMC

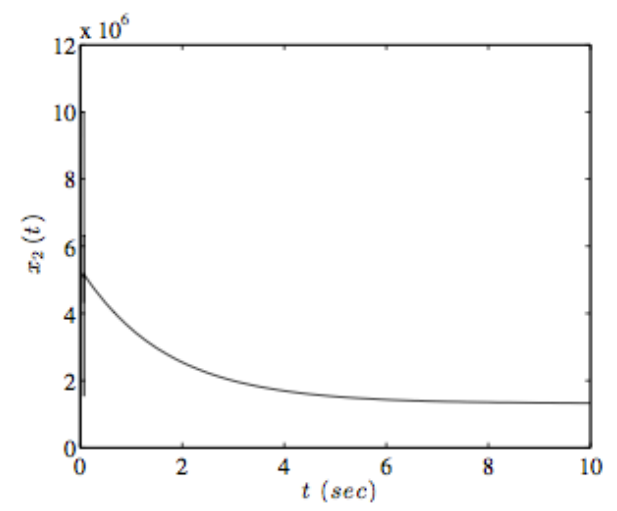

Figure 6. The Load Pressure $x_{2}(t)$ when Using the SDRE

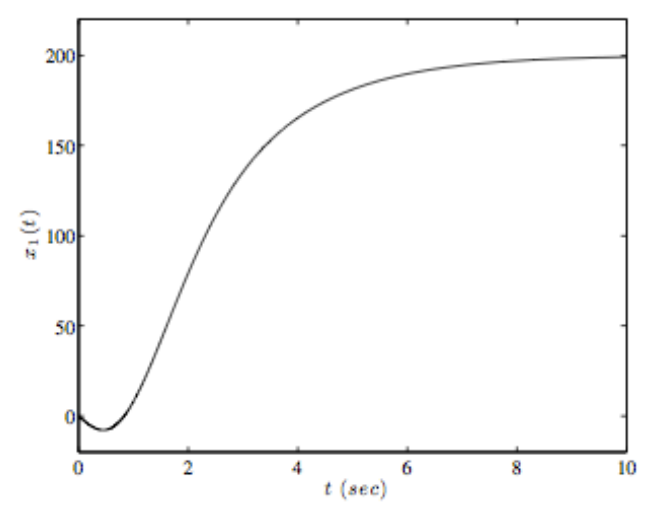

Figure 8. The Output Velocity $x_{1}(t)$ when Using Dynamic SMC under Perturbation

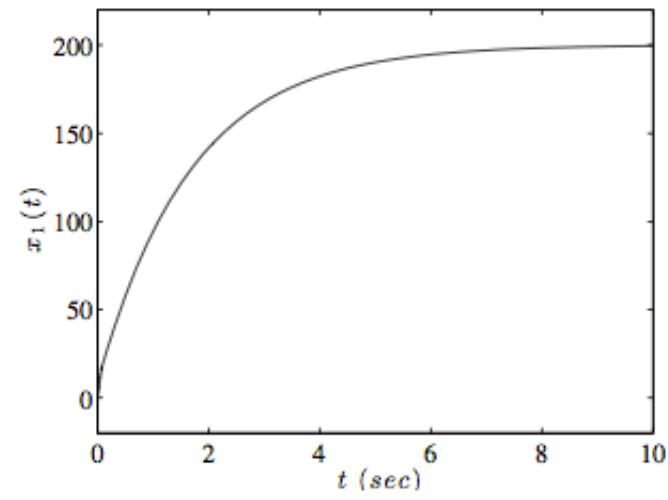

Figure 5. The Motor Velocity $x_{1}(t)$ when Using the SDRE

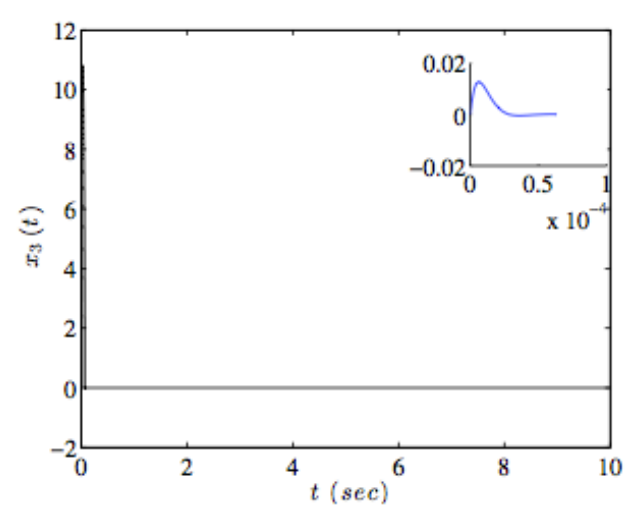

Figure 7. The Valve Displacement $x_{3}(t)$ when Using the SDRE

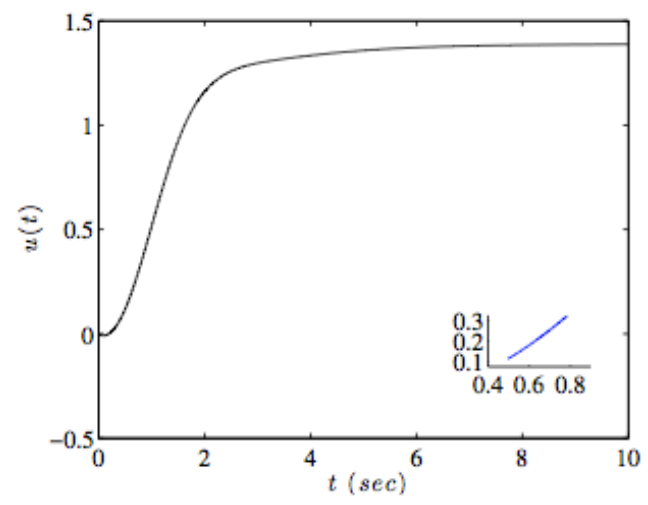

Figure 9. The Dynamic SMC under Perturbation 


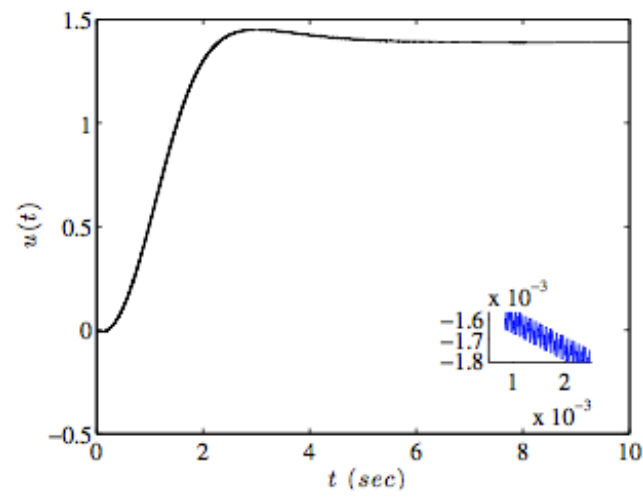
Figure 10. Chattering Behavior in
the Classical SMC Law

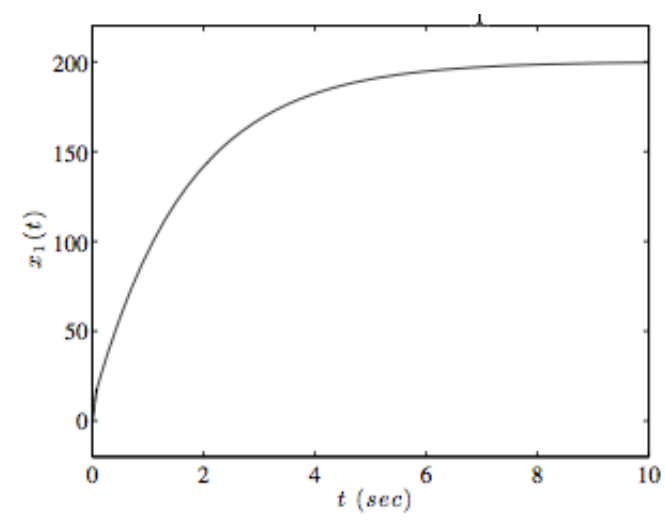

Figure 11. The Output Velocity $\mathrm{x}_{1}(\mathrm{t})$ when Using the SDRE Controller

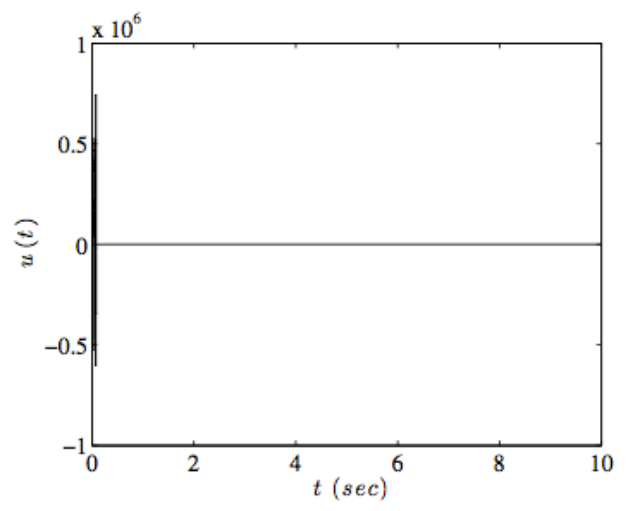

Figure 12. The SDRE Controller under Perturbation

\section{References}

[1] F. D. Norvelle, "Electrohydraulic Control Systems", Prentice-Hall, Inc., (2000).

[2] R. B. Walters, "Hydraulic and Electrohydraulic Control Systems", Elsevier Science Publishing co., Inc., (1991).

[3] M. E. Rabi, "Fluid Power Engineering", McGraw-Hill Companies Inc., (2009).

[4] Jovanovic, M., "Nonlinear control of an electrohydraulic velocity servosystem," Proceedings of the 2002 American Control Conference, 2002 May 8-10, USA.

[5] C. Kaddissi, J-P. Kenne and M. Saad, "IEEE Transactions on Mechatronics", vol. 16, no. 16, (2011).

[6] H. A. Mintsa, R. Venugopal, J-P. Kenne and C. Belleau, "IEEE Tran. Control Systems Tech.", vol. 20, no. 4 , (2012).

[7] M. A. Shoorehdeli, M. Teshnehalab and H. A. Shoorehdeli, "Velocity Control of an Electro Hydraulic Servosystem, IEEE Int. Conference on Systems, Man and Cybernetics, (2007) October 7-10, Montreal, Canada.

[8] G. Hasanifard, M. H. Zarif and A. A. Ghareveisi, "Nonlinear Robust Backstepping Control of an Electrohydraulic Velocity Servosystem", Mediterranean Conference on Control and Automation, (2007) June 27-29, Athens.

[9] D. Garagic and K. Srinivasan, "IEEE Tran. Control Systems Tech.", vol. 12, no. 2 (2004)

[10] G. Wu, N. Sepehri and L. Ziaei, "IEE Proceedings-Control Theory and Applications", vol. 145, no. 5 (1998)

[11] C-L. Hwang, "IEEE/ASME Transactions on Mechatronics", vol. 4, no. 1, (1999).

[12] O. Cerman and P. Husek, "Expert Systems with Applications", vol. 39, (2012).

[13] K. D. Young, V. I. Utkin and U. Ozguner, "IEEE Transactions on Control Systems Tech.”, vol. 7, no. 3 (1999).

[14] E. B. Erdem and A. G. Alleyne, "IEEE Transactions on Control Systems Tech.", vol. 12, no. 1, (2004).

[15] T. Cimen, "Annual Reviews in Control”, vol. 34, (2010).

[16] A. R. Z. Bidaki, M. Malboubi, M. Jahanshahi, R. Pirmoradi, M. Reza and A. A. Abad, "Australian Journal of Basic and Applied Sciences", vol. 5, no. 12, (2011). 
[17] H. Sira-Ramirez, O. Llanes-Santiago and N. A. Fernandez, "International Journal of Robust and Nonlinear Control", vol. 6, (1996).

[18] J-L. Chang, "Journal of Control Science and Engineering”, vol. 2012, Article ID 564906 (2012).

[19] A. J. Koshkouei, K. J. Burnham and A. S. I. Zinober, "IEE Proceedings - Control Theory and Applications", vol. 152, no. 4, (2005).

[20] S. R. Nekoo, "ISA Transactions", vol. 52, no. 2, (2013).

[21] T. Cimen, "State-Dependent Riccati Equation (SDRE) Control: A Survey", Proc. of the $17^{\text {th }}$ IFAC World Congress, (2008) July 6-11, South Korea.
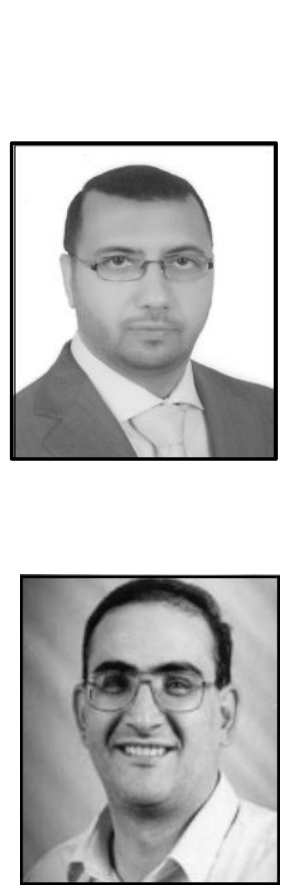

\section{Authors}

Saleh Alshamali, he obtained his B.S. degree in Electrical \& Computer Engineering in 1997 from the University of Missouri-Columbia, USA. He received the M.S. degree and the Ph.D. degree in Electrical Engineering in 2000 and 2004 from the University of Florida, Gainesville, USA. He is currently an Assistant Professor in the Electrical Engineering Department at Kuwait University. His research interests include stability of nonlinear systems, control design for linear and nonlinear systems, and control applications.

Mohamed Zribi, he obtained the B.S. degree in Electrical Engineering (Magna Cum Laude Honors) in 1985 from the University of Houston, Houston, Texas, USA. He received the M.S.E.E Degree and the Ph.D. Degree in Electrical Engineering in 1987 and 1992 from Purdue University, West Lafayette, Indiana, USA. He held a faculty position from 1992 till 1998 in the School of Electrical Engineering, Nanyang Technological University, Singapore. $\mathrm{He}$ is currently a Professor in the Electrical Engineering Department, College of Engineering Petroleum, Kuwait University. His research interests are in nonlinear control, adaptive control, control applications and robotics. 